\title{
The Effect of Perinatal Maternal Health Knowledge Explaining Combined Full-time Nursing Staff Accompanying on Maternal Labor and Pregnancy
}

\author{
Liang Wenfei, Zuo Li, Gao Yujing, Huang Xinke* \\ Department of Gynecology and Obstetrics, the First Affiliated Hospital of Jinan University, Guangzhou, China \\ Email address: \\ 929901838@qq.com (Huang Xinke) \\ *Corresponding author \\ To cite this article: \\ Liang Wenfei, Zuo Li, Gao Yujing, Huang Xinke. The Effect of Perinatal Maternal Health Knowledge Explaining Combined Full-time \\ Nursing Staff Accompanying on Maternal Labor and Pregnancy. Journal of Gynecology and Obstetrics. Vol. 7, No. 5, 2019 , pp. 138-141. \\ doi: $10.11648 /$ j.jgo.20190705.13
}

Received: August 29, 2019; Accepted: September 16, 2019; Published: September 30, 2019

\begin{abstract}
Objective: TO investigate the effect of perinatal maternal health knowledge explaining combined full-time nursing staff accompanying on maternal labor and pregnancy. Methods In our hospital from January 2016 to October 2018, 173 primiparas were randomly divided into control group (87 cases) and nursing group (86 cases). The control group was given perinatal routine care, the observation group was given perinatal maternal health knowledge explaining combined full-time nursing staff accompanying. The maternal psychological state, labor, pregnancy outcome and neonatal Apgar score were compared between the two groups. Results: Before the intervention, the scores of SAS (51.37 \pm 5.73$)$ and SDS (63.32 \pm 6.17$)$ in the nursing group were not significantly different from those in the control group (SAS $51.74 \pm 5.82$ and SDS 63.47 \pm 6.21$)(\mathrm{P}>0.05)$. After the intervention, the SAS (37.51 \pm 3.51 points) and SDS (44.58 \pm 4.23 points) scores in the observation group were significantly lower than those control group (43.36 \pm 4.95 points, $52.46 \pm 4.51$ points $)(\mathrm{P}<0.05)$. Besides, there was no significant difference between the nursing group (401.3 $\pm 102.6 \mathrm{~min})$ and the control group (404.2 $\pm 104.5 \mathrm{~min})$ in the first stage of labor $(P>0.05)$. However, the second $(25.2 \pm 4.3 \mathrm{~min})$ and third $(8.3 \pm 2.1 \mathrm{~min})$ labor time of nursing group was significantly less than those of the control group $(46.8 \pm 6.7 \mathrm{~min}, 11.4 \pm 2.6 \mathrm{~min})(P<0.05)$. And, the pregnancy outcomes of the nursing group were significantly better than the control group $(P<0.05)$. Furthermore, the Apgar score in the nursing group $(9.25 \pm 1.03)$ was significantly higher than that in the control group $(8.24 \pm 1.35)(P<0.05)$. Conclusion: Perinatal maternal health knowledge and combined with full-time nursing staff can help reduce maternal anxiety and depression, shorten labor time, improve maternal pregnancy outcomes, and improve neonatal Apgar score, which is worthy of clinical application.
\end{abstract}

Keywords: Perinatal Maternal Health Knowledge, Full-time Nursing Staff Accompanying, Labor Process, Pregnancy Outcome

\section{Introduction}

Due to the lack of obstetrics related knowledge and severe pain during delivery, parturient tends to cause a strong degree of anxiety and depression, and the incidence is as high as $16 \%$ [1]. Severe anxiety can cause certain obstacles in the process of delivery, which causes the parturient to not cooperate with the guidance of the doctor [2]. As a result, the process of labor is too long, the rate of cesarean section is increased, the fetal distress is in the uterus, and even the life of the parturient and the fetus is dangerous. Therefore, how to make parturients cooperate with medical staff in the process of delivery, to reduce the occurrence of adverse events has been the focus of clinical attention [3]. It plays an important role in improving the delivery process of parturients by explaining the health knowledge of parturient pregnancy in various ways [4, 5]. Total labor nursing staff accompanying refers to the maternal delivery process has been accompanied by medical staff, so that parturients feel the sense of responsibility of medical staff. When you need help, you can find an assistant at any time, and obtain professional and detailed guidance for the whole production process. In this study, we explained the knowledge 
of parturient maternal health and accompanied the nursing staff during labor. The results showed that this method could improve the psychological state of parturients and shorten the delivery process.

\section{Methods}

\subsection{General Data}

From January 2016 to October 2018, 173 patients of first-time parturients were selected according to inclusion and exclusion criteria in The First A liated Hospital of Jinan University. Among them, 86 cases were included in the control group and 87 cases were included in the nursing group. In the control group, the age of the patients was 22 - 32 years old, the gestational age was 26 - 33 weeks and height of the patients was $154-168 \mathrm{~cm}$. In the nursing group, the age of the patients, gestational age and the height were similar. Inclusion criteria: puerpera has good communication skills; understand the research process and willing to cooperate with the research; the maternal cycle was more than 4 weeks. Both groups were single pregnancies. Exclusion criteria: mental disorder; complicated with serious organ disease; complicated with endocrine system disease. There was no significant difference in general data between the two groups $(P>0.05)$. (Table 1)

Table 1. General data of two groups.

\begin{tabular}{llllll}
\hline \multirow{2}{*}{ Group } & Age (year) & Gestational age (week) & Height (cm) & \multicolumn{2}{c}{ Education } \\
\cline { 4 - 6 } & & & $158.31 \pm 5.06$ & 18 & middle school \\
\hline Control $(\mathrm{n}=87)$ & $27.42 \pm 4.31$ & $34.36 \pm 4.17$ & $159.06 \pm 4.98$ & 19 & 24 \\
Nursing $(\mathrm{n}=86)$ & $27.26 \pm 4.28$ & $34.29 \pm 4.24$ & 0.218 & 0.866 & 26 \\
$P$ & 0.423 & 0.387 & 41 & \\
\hline
\end{tabular}

Note: Middle school means that the level of education is middle education or lower than that of middle school.

High school means that the level of education is middle education. College or above means that the level of education is university education or higher than university education.

\subsection{Nursing Method}

The control group was given perinatal routine nursing care, and the nursing group was given perinatal health knowledge explanation combined with whole-process nursing care. A perinatal health knowledge presentation team was established in our hospital, including 2 deputy chief nurses, 2 in charge nurses, 2 nurses and 6 team members. Perinatal health knowledge presentation includes formulate health guidance content, multimedia health education, explain perinatal nutrition, nursing knowledge, prenatal signs and delivery process.

Nursing staff accompanying during the whole process of labor: a nurse accompanies the puerpera during the whole process of labor. It provides a warm environment for puerpera to stay in the hospital, including caring about puerpera, gaining puerpera's trust with professional knowledge, making puerpera fully prepared, informing puerpera of these normal phenomena and reduce puerpera anxiety.

\subsection{Observational Indexes}

The observed indicators include the self-rating anxiety scale, the self-rating depression scale, 3 labor cycles, pregnancy outcome, postpartum hemorrhage and Apgar score for neonates. Labor needs to evaluate 3 labor cycles. Pregnancy outcome include cesarean section, premature delivery, fetal distress, neonatal asphyxia and postpartum hemorrhage.

Psychological status: the self-rating anxiety scale (SAS) was used to evaluate the anxiety level of patients [6]. The scale includes 20 items, 15 of which are positive and 5 of which are negative. Each project is divided into 4 grades. The highest score was 100 points, and the lowest score was 25 points. The self-rating depression scale (SDS) was used as the SDS rating scale with 20 items, 15 of which were positive and 5 were negative [7].

Apgar score for neonates: The Apgar score for neonates includes muscle tension, respiration, pulse and frowning stimulation. The Apgar score for neonates is normal with a maximum score of 10 , moderate asphyxia with a maximum score of 7-10, mild asphyxia with a maximum score of 4-7, and severe asphyxia with a maximum score of less than 4 [8].

\subsection{Statistical Analysis}

The data of 163 patients were analyzed by SPSS20.0 software. Measurement data are presented as mean \pm standard deviation (SD). Enumeration data were evaluated with the chi-square test and categorical data were compared by the Wilcoxon signed-rank test. And P-value of $<0.05$ was considered statistically significant.

\section{Results}

\subsection{Comparison of Maternal Psychological Status Between the Two Groups}

Before the intervention, there was no significant difference in SAS $(51.37 \pm 5.73$ points $)$ and SDS (63.32 \pm 6.17 points) scores between the nursing group and the control group $(51.74 \pm 5.82$ points, $63.47 \pm 6.21$ points $)$ $(\mathrm{P}>0.05)$. After intervention, the scores of SAS (37.51 \pm 3.51 points) and SDS (44.58 \pm 4.23 points) in the nursing group were significantly lower than those in the control group $(43.36 \pm 4.95$ points, $52.46 \pm 4.51$ points) $(\mathrm{P}<0.05)$. (Table 2) 
Table 2. Comparison of psychological states between the two groups $(\bar{x} \pm s)$.

\begin{tabular}{llllll}
\hline \multirow{2}{*}{ Group } & \multirow{2}{*}{$\mathbf{n}$} & \multicolumn{2}{l}{ SAS (score) intervention } & \multicolumn{2}{l}{ SDS (score) intervention } \\
\cline { 3 - 6 } & & Before & After & Before & After \\
\hline Control & 87 & $51.37 \pm 5.73$ & $37.51 \pm 3.51^{*}$ & $63.32 \pm 6.17$ & $44.58 \pm 4.23^{*}$ \\
Nursing & 86 & $51.74 \pm 5.82$ & $43.36 \pm 4.95^{*}$ & $63.47 \pm 6.21$ & $52.46 \pm 4.51^{*}$ \\
$\mathrm{t}$ & & 0.936 & 5.417 & 1.031 & 6.045 \\
$P$ & & 0.192 & 0.008 & 0.104 & 0.003 \\
\hline
\end{tabular}

Note: *, significantly different between pre-intervention and pose-intervention, $P<0.05$. The difference was significantly different compare to two groups after the intervention, $P<0.05$.

\subsection{Comparison of Labor Stages Between the Two Groups}

There was no significant difference between the nursing group ( $401.3 \pm 102.6 \mathrm{~min})$ and the control group $(404.2 \pm 104.5 \mathrm{~min})$ in the first labor duration $(P>0.05)$. The duration of labor in the nursing group $(25.2 \pm 4.3 \mathrm{~min})$ and the third $(8.3 \pm 2.1 \mathrm{~min})$ was significantly shorter than that in the control group $(46.8 \pm 6.7 \mathrm{~min}, 11.4 \pm 2.6 \mathrm{~min})(P<0.05)$. (Table 3$)$

Table 3. Comparison of labor stages between the two groups [( $\bar{x} \pm s), \min ]$.

\begin{tabular}{lllll}
\hline Group & n & First labor & Second labor & Third labor \\
\hline Control & 87 & $401.3 \pm 102.6$ & $25.2 \pm 4.3$ & $8.3 \pm 2.1$ \\
Nursing & 86 & $404.2 \pm 104.5$ & $46.8 \pm 6.7$ & $11.4 \pm 2.6$ \\
$t$ & & 0.863 & 9.257 & 4.624 \\
$P$ & & 0.247 & 0.00 & 0.012 \\
\hline
\end{tabular}

\subsection{Comparison of Pregnancy Outcomes Between the Two Groups}

The pregnancy outcome of the nursing group was significantly better than that of the control group $(P<0.05)$. (Table 4$)$

Table 4. Comparison of pregnancy outcomes between the two groups (n, \%).

\begin{tabular}{llllll}
\hline Group & Cesarean delivery & Preterm birth & Fetal distress & Neonatal asphyxia & Postpartum hemorrhage \\
\hline Control $(\mathrm{n}=86)$ & $5(5.7)$ & $3(3.4)$ & $2(2.3)$ & $1(1.1)$ & $2(2.3)$ \\
Nursing $(\mathrm{n}=87)$ & $40(46.5)$ & $15(17.4)$ & $9(10.5)$ & $7(81.4)$ & $13(15.1)$ \\
$\mathrm{X}^{2}$ & 20.877 & 9.085 & 4.844 & 4.791 & 8.973 \\
$P$ & 0.000 & 0.003 & 0.028 & 0.029 & 0.003 \\
\hline
\end{tabular}

\subsection{Comparison of Apgar Scores Between the Two Groups}

The neonatal Apgar score of the nursing group $(9.25 \pm 1.03)$ was significantly higher than that of the control group (8.24 $\pm 1.35)(P<0.05)$.

\section{Discussion}

Delivery is a normal physiological process for women, but due to the lack of knowledge of delivery, pain and poor medical conditions, delivery does cause life danger to parturient. There are often many psychological problems in the process of delivery, and will have a negative impact on the pregnancy outcome of parturient [9-11]. With the development of modern medical technology, people's health consciousness has been gradually improved. Perinatal training is initiated in the early stages of pregnancy, and regular examinations are conducted to eliminate adverse factors to both the fetus and the pregnant woman. The explanation of perinatal pregnancy health knowledge is transmitted to parturient women through multiple channels to ensure that they can fully understand the knowledge of parturient women. Based on humanistic care, nursing staff throughout the labor process consider the needs of puerpera from different aspects to ensure that puerpera can meet the psychological, physiological and life needs in the production process [12].

This study used perinatal pregnancy health knowledge to explain the nursing of first time pregnant women in combination with nursing staff throughout labor. The results showed that the mental state of the nursing group was significantly better than that of the control group. It suggests that teaching perinatal health knowledge combined with whole-process nursing care and routine perinatal care can improve maternal psychological state [13]. The whole process nurses accompany puerperas' psychology to play the consolation function. What is more important is that the puerpera in the process of labor by the nursing staff to pay close attention to ensure the safety of puerpera. In the nursing group, the second and third stages of labor were significantly shorter than those in the control group, suggesting that perinatal pregnancy health knowledge explanation combined with nursing staff in the whole labor process was beneficial to shorten the length of labor. It may be related to maternal knowledge of childbirth and nursing staff's fine guidance and psychological comfort [13]. The pregnancy outcome of the nursing group was significantly better than that of the control group. The main reason is that maternal knowledge and professional guidance of nursing staff promote the smooth delivery of maternal [14]. Therefore, perinatal pregnancy health knowledge in combination with the whole process of nursing care is very helpful. Besides, the Apgar score of 
neonates in the nursing group was significantly higher than that in the control group. This fully reflects that perinatal pregnancy health knowledge interpretation combined with whole-labor nursing staff is more conducive to newborn health [15]. This makes us understand that health guidance in diet, exercise and regular physical examination, as well as good cooperation with medical staff in the production process, is of great significance to both mothers and newborns. However, due to time constraints, this study did not study in depth the mechanism of obvious improvement of maternal pregnancy outcome, and the research team will continue to pay attention to reports on childbirth, so that provides more reliable evidence for clinical practice.

\section{Conclusion}

In conclusion, perinatal maternal health knowledge and combined with full-time nursing can reduce maternal anxiety and depression, shorten labor time, improve maternal pregnancy outcomes, and improve neonatal Apgar score, which is worthy of clinical application.

\section{References}

[1] De Carli P, Costantini I, Sessa P, et al. The expectant social mind: A systematic review of face processing during pregnancy and the effect of depression and anxiety. Neuroscience and biobehavioral reviews. 2019; 102: 153-71.

[2] Gjerde LC, Eilertsen EM, Reichborn-Kjennerud T, et al. Maternal perinatal and concurrent depressive symptoms and child behavior problems: a sibling comparison study. Journal of child psychology and psychiatry, and allied disciplines. 2017; 58 (7): 779-86.

[3] Rutherford JN, Asiodu IV, Liese KL. Reintegrating modern birth practice within ancient birth process: What high cesarean rates ignore about physiologic birth. Am J Hum Biol. 2019; 31 (2).

[4] Yee LM, Caughey AB, Grobman WA, Cheng YW. Is advanced maternal age associated with adverse perinatal outcomes among women with twin gestations? Am J Obstet Gynecol. 2018; 218 (1): S102-S3.
[5] Fuchs F, Monet B, Ducruet T, et al. Effect of maternal age on the risk of preterm birth: A large cohort study. PloS one. 2018; $13(1)$.

[6] Ma XM, Wang $\mathrm{Y}, \mathrm{Hu} \mathrm{H}$, et al. The impact of resilience on prenatal anxiety and depression among pregnant women in Shanghai. J Affect Disorders. 2019; 250: 57-64.

[7] Zhang JY, Cui YX, Zhou YQ, Li YL. Effects of mindfulness-based stress reduction on prenatal stress, anxiety and depression. Psychol Health Med. 2019; 24 (1): 51-8.

[8] Towers CV, Compton S, Chattin K, et al. Neonatal Outcome Following Delivery with a 1-Minute Apgar Score of 1, 2, or 3 Followed by an Apgar Score of $>=7$ at 5 Minutes. J Reprod Med. 2018; 63 (11-12): 501-4.

[9] Valentine G, Chu DM, Stewart CJ, Aagaard KM. Relationships Between Perinatal Interventions, Maternal-Infant Microbiomes, and Neonatal Outcomes. Clin Perinatol. 2018; 45 (2): 339-+.

[10] Kougias DG, Cortes LR, Moody L, et al. Effects of Perinatal Exposure to Phthalates and a High-Fat Diet on Maternal Behavior and Pup Development and Social Play. Endocrinology. 2018; 159 (2): 1088-105.

[11] Sosinsky AZ, Freeman MP, Savella GM, et al. Delusional Pregnancy Presenting to the Massachusetts General Hospital National Pregnancy Registry for Atypical Antipsychotics. Journal of clinical psychopharmacology. 2017; 37 (4): 472-4.

[12] Henderson ZT, Ernst K, Simpson KR, et al. The National Network of State Perinatal Quality Collaboratives: A Growing Movement to Improve Maternal and Infant Health. Journal of women's health. 2018; 27 (3): 221-6.

[13] Katon JG, Lewis L, Hercinovic S, et al. Improving Perinatal Mental Health Care for Women Veterans: Description of a Quality Improvement Program. Matern Child Hlth J. 2017; 21 (8): 1598-605.

[14] Gleddie M, Stahlke S, Paul P. Nurses' perceptions of the dynamics and impacts of teamwork with physicians in labour and delivery. Journal of interprofessional care. 2018: 1-11.

[15] Ayiasi RM, Criel B, Orach CG, et al. Primary healthcare worker knowledge related to prenatal and immediate newborn care: a cross sectional study in Masindi, Uganda. Bmc Health Serv Res. 2014; 14. 